\title{
Application of Music as an Element in Art Therapy, on Cancer Patients, Leads to Emotional Conflict Resolution and Body Healing, with an Insight into Sufi Music, Ancient Music Therapies, their Healing History as Well as Properties
}

\author{
Spriha Mukherjee ${ }^{1 *}$
}

Keywords: Music, Art Therapy, Cancer Patients, Emotional Conflict, Body Healing, Sufi Music, Ancient Music Therapies

The Universe created a magical spell which not only held the ability to heal and enhance the health of the mankind but also add most pleasant rhythm to it. This unique creation of universe was termed as "Music".

Music has been observed to hold immense importance in lives of human being from the time of its inception. It turned out to be a blessing in the attire of a magnificent form of an art. Music held ground in man's life in various ways, such as for its ability to heal, entertain, means of communication, expression of emotions etc. It has been found to add significant changes in the way a society works. In early times music was used as a means of communication, people would play drums or any other instruments to collect or gather crowd with the purpose of conveying any message. During events and festivals it was regarded to be a pious act to play musical instruments, not only from the point of view of entertainment but also it was based on the belief that music purifies the vibration in any particular environment. Even it has been used to symbolize various emotions during early ages.

Music has been of immense importance in lives of mankind in many ways, but what has made it distinctive from other sources of communication or entertainments were its properties of healing. Yes it sounds unbelievable but is a proven fact since times of Vedic period till now, that music does hold the ability to heal. And surprisingly its property of healing was not restricted to just emotions and mind, but its magical properties extended upto various bodily diseases as well. Nature had its own way of blessing human beings with the finest of its creations and inventions, and music did turn out to be such a blessing in real times. Since early ages till date, various researchers, musicians, therapists and experts have been able to study, work, analyze and finally

\footnotetext{
${ }^{1}$ Psychologist, Academic Counsellor, SGT University, Gurgaon, India

*Responding Author

(c) 2016 I S Mukherjee; licensee IJIP. This is an Open Access Research distributed under the terms of the Creative Commons Attribution License (http://creativecommons.org/licenses/by/2.0), which permits unrestricted use, distribution, and reproduction in any Medium, provided the original work is properly cited.
} 
understand the wonders that music can perform in an individual's life. And there efforts have always paid successful results and they were able to discover more and better aspects of using music as an aid of human's wellbeing.

Continuous efforts and researches eventually lead to the invention of music therapies. Music therapy can be defined and understood as a process of treating a patient by means of a combination of musical notes in the presence of a trained therapist or someone who holds ample knowledge about the same. When an initiation to self exploration, self- assessment and selfanalysis takes place on part of the client, by means of music, thus resulting into self satisfaction and overall enhancement, but under the apt guidance of a therapist or an individual trained in the given field, this entire process is known as Music therapy sessions.

In any music therapy session, an individual is made to hear some specific ragas or musical notes as may be required for the purpose of healing. Music therapy is an economic therapy involving least cost expenses. Thus it is available the most preferred therapy among people as well.

The neurological and biological effects of music has not been taken into consideration at first but with time and examples that proved its healing abilities, it has now gained grounds in the medical field and soon might become one of the conventional therapies as well. Many therapists are now a day's using music therapy as a complementary and alternative medicine for treating patients with varied diseases. Music has shown its contributions in various areas like, spiritual transformation, education, entertainment, treatment etc. Music has been found to have the ability to energize individual, inbuilt positive emotions in him/her and act as a support in times of stress and in the process of enhancing wellbeing. Many people use music as a defense mechanism for coping up from stressful situations. Listening to music helped them reduce their level of stress and displace their anger and negativity in a more constructive manner.

Indian music itself has been regarded as a blessing for a soul, for it has the ability to purify an individual from within as and when the different combinations of musical notes, popularly known as Swaras, are heard. Indians thus believe that music should always form a part of any ritual, event or celebration. In fact they held strong faith in its healing abilities. Different cultures and religion in India relates their culture to music in different ways. Like in some cultures use of "Dhol", "nagada" or "Dhaak" is made,some use "shenai" and some use instruments like violin, harp etc.

With changing trends, the use of various musical instruments and their usage has been changing. People now look forward to play and listen to music serving multiple purposes. And thus we get to observe its effective applications in various fields. The healing properties of music might not be the only reason behind its increasing demand in the present scenario but definitely is an essential cause. Music is no longer a noise to those who understands its relevance in the effective 
daily functioning of life. It doesn't only serve as a treat to their ears but also enhances their ability to connect with their lives.

Art therapy as the name suggests, is a process of self exploration, self analysis and understanding of self, via medium of art and under the supervision of a specialized art therapist. In the process of art therapy, an individual gets to attain an opportunity to heal oneself mentally, physically and emotionally, thereby enhancing the level of overall wellbeing. Life is not a cake walk for any individual. It is a journey of experiences and learning comprising of various expected as well as unexpected elements. All that is needed is the willingness of an individual to embrace all that comes his/her way with the spirit of living it to the fullest. Art therapy has been observed of being a medium, which can be used for managing, handling, sorting, balancing and maintaining various behavioral and emotional patterns. This therapy involves no extra efforts on part of the performing client and hence holds an edge over other therapies for its usage, applicability, feasibility reliability and validity.

Music therapy in combination with art therapy when applied on patients with various health issues, have been observed to cause better healing in comparison to other therapies. Patient or the client is able perform at their comfort, involving no stress or added on efforts. They get the privilege to express freely what they feel or think via an art form and hence are able to creatively went out their emotions, as well as channelize their thought pattern. Such expressivity, channelization and management of thoughts enhance the level of resilience (the ability of an individual to cope from a difficult situation or an event), self esteem and wellbeing.

There are cases wherein patients were treated with art therapy in combination with music therapy and have responded better over the period of time. Since art therapy is all about free-hand creativity used for the purpose of healing, involving no artistic efficiency, and music therapy helps in relaxing the state of mind of an individual thereby increasing the level of concentration and focus; they make an excellent combination for over all internal healing.

\section{Effect of Art and Music Therapy on Cancer Patients:}

Patients suffering from cancer experience a lot of emotional and physical toll, they face immense difficulty in accepting their problem. On getting to realize their health issues, accepting and coping with it becomes a struggle in itself. Here is where the role of Art and Music therapy comes into picture easing the process of expressivity of emotions like anger, stress and sadness.

Art therapy helps in expressivity of emotions in a way that the patient is able to express all that he/she would be wanting to, but feels hesitant somehow to do so. Via medium of art a patient is able to give their emotions a form which in turn eases the analysis of these emotions on a better platform, thereby enabling the process of conflict resolution. The emotional toll existing within a 
Application of Music as an Element in Art Therapy, on Cancer Patients, Leads to Emotional Conflict Resolution and Body Healing. With an Insight into Sufi Music, Ancient Music Therapies, their Healing History as Well as Properties

cancer patient leads to a lot of internal conflicts, resolution of which becomes necessary for initiating the process of healing.

Music therapy compliments the process of art therapy and this is the reason why they make an excellent combination to be applied on the cancer patients for initiating their healing process. Musical notes carry vibrations which when hits the mind affects the thought pattern of the individual, thereby leading to an overall thought level enhancement and mental peace.

Music therapy when applied as an added element in art therapy on the cancer patients, there are various positive outcomes which are observed like; the right hemisphere gets strengthened, the immunity of the body increases and repetitive application leads to an overall development of the body functionality.

\section{MUSIC THERAPY IN RELATION TO CANCER HEALING}

Bailey (1983) found that those patients who listened to live music showed less of tension and anxiety and more of vigor than those who listened to tape recorded music. Human element inherent in live music was considered to be important, and of great use for the purpose of relieving tension and promoting vigor. Hence use of live music was taken into high consideration in the process of healing.

Lindquist Bonny (1986) found that as compared to traditional beliefs, more recent ascendance shows a change that has been created in the attitude of the practitioners who now believe music therapy as a healing mode at a greater position of acceptance. Their further researches studied the various types of music which can be brought in use as a part of the therapies, for ultimately enhancing the level of healing among the patients.

Cook and Janet D. (1986) found that music holds many qualities and as an intervention can be used in the process of coping among cancer patients. Music was found to stand as a nonthreatening form of communication, which holds the capability to break down all linguistic, educational, cultural and emotional barriers. Thus it serves as a mode of eliciting feelings, emotions and thoughts thereby serving as an outlet for emotional healing among patients during the process.

Jhonston, Davis and Jacqueline (1996) found that music therapy can act as a noninvasive holistic approach for bridging the gap between oncology patients and intensive care units. Intensive care units are stressful and frightening, thus introducing music therapy in such an environment in accordance with the requirement makes it comfortable for the patients to adjust themselves to such an environment. 
Burns SJ, Harbuz MS,Hucklebridge F,Blunt L ( 2001) observed that there were altered energy levels in both interventions as well as an increase in the level of well-being and relaxation. During the listening experience it was observed that there was an increase in their level of immunoglobulin A and decrease in their cortisol, in the two day period. It ultimately leads to the development of a link between their positive emotion and enhanced cancer healing.

Burns (2001) found that often patients suffer from continuous and elevated level of distress after their cancer treatment. This negatively affects their endocrine system and decreases their quality of life. In such cases the use of guided imaginary and music (GIM) has been found to have made a significant contribution in improving the quality of life. It was observed that GIM sessions lead to better mood scores as well as quality of life scores.

Ernst (2001) found that complementary medication has gained grounds in the healing of palliative cancer care. This study was done with an aim to guide the clinicians about some commonly used complementary therapies, including music therapy. Results showed that there has been an increasing demand for the use of music therapy along with other complementary and alternative medicines for the purpose of healing palliative cancer patients.

Waldon (2001) found that there are significant improvement in mood states scores when elderly patients of onchology where made to undergo music therapy sessions. Improvements were found from pre-session levels to post-sessions level. The extents of improvements were analyzed at each level and accordingly comparisons were made for better understanding of the results.

Callanghan and McDermott ( 2004) found that where life's vulnerability is constantly apparent among patients, affirmed their aliveness resonating with an expanded consciousness, as a result of affective, contemplative and imagined moments in music therapy. Relevance of music in human life from the point of view of healing and wellbeing enhancement has been to a great extent.

Dayklin (2006) found that there are different types of music and music therapies which contributes differently in the process of cancer healing. He also studied the provider's point of view. There are a few challenges that are faced by the music therapist with the changing world of cancer, development in the level of treatment and organization of care givers and supportive care practitioners.

Clark, Downton, Wells, Frazier, Eck, Hepworth and Chakravarthy (2006) found that music listening interventions brought down level of emotional distress and symptoms among patients who were undergoing curative radiation therapy. Emotional distress comprised of their anxiety, 
Application of Music as an Element in Art Therapy, on Cancer Patients, Leads to Emotional Conflict Resolution and Body Healing. With an Insight into Sufi Music, Ancient Music Therapies, their Healing History as Well as Properties

depression and treatment related distress. Patients who listened to self selected music were observed with lower level of anxiety and treatment related distress.

Stuart McLean, Leslie Bunt (2007) found that music therapy as a part of complementary and alternative medicine acts as a supportive unit in cancer care and treatment. It has been found to be making a distinctive contribution as a creative therapy. Analyses show that importance of identity and role of creativity in the process of individuation emerge to be the key themes in as a part of the therapy.

Mei Li, Yan, Zhou, Dang, Whang and Zhang (2010) found that followed by radical mastectomy, music therapy contributes to the positive affects of alleviating pain among the breast cancer patients. The intervention group agreed to undergo music therapy sessions after their radical mastectomy in addition to their routine nursing care. Their pain scores were then measured by general questionnaire and Chinese short-form of McGill pain Questionnaire, at a baseline. Music therapy was found to reduce the pain rating index score among the intervention group.

Olofsson and Fosson (2011) found that there has to be coordination among the providers of music therapy in order to derive maximum benefits from its application in the healing sessions. There has to be interdisciplinary cooperation between nurses and music therapist on clinical and educational level for effective results. Concept and knowledge of music therapy has to be brought in coordination with the scientific orientation for the purpose of its better application leading to better results.

Stanczyk (2011) found that cancer disrupts, physical, emotional and social well-being of an individual patient suffering from it. By using music therapy as a part of complementary medicine programme, benefits patient in healing of range of emotions such as anger, depression, guilt, embarrassment, shame etc.Both interactive music therapy techniques ( involving singing, instrumental improvisation) as well as receptive music therapy techniques (involving listening to imaginary music, live recorded music) contributed in improving the mood levels as well as decreasing the level of anxiety, depression, stress and pain among the patients.

\section{MUSIC THERAPY AND BODY HEALING}

Cheryl and Godley (1987) found that music, relaxation and imagery strengthens the right hemisphere of the brain thus influences the immune system of the body, enhances positive attitude towards health and directs a healing process within the body. Thus music therapy has been of immense help in process of rehabilitation as well. 
Updike, Phyllis, Charles and David (1987) found that listening to music affect desirable pattern shifts in physiological and emotional states of patients awaiting an elective plastic surgery procedure. The emotional responses were evaluated by an open-ended, nondirective questionnaire. The questionnaire was developed around 5 categories of depression, despair and sadness, psychological isolation and defensiveness; anxiety; preoccupation with pain and difficulty in medical management.

Moss (1988) found that sedative music intervention proved to be effective in reducing sympathetic nervous system activity. It also reduces situational or state anxiety in association with the surgery. Music produced by instruments like harp, lyre or flute can be used for healing the sick and enhancing a healthy life.

Mckinney (1990) found that music therapy had no effect on the number of senses, vividness of the imagery, type of imagery, activity of the imagery and the percentage of time the subject was distracted or imaging. But music therapy did play a role in increasing the intensity of feelings experienced. Hence music therapy is used for the treatment of physical disease as well as in psychotherapy.

Cowan (1991) found how music helps in the reduction of anxiety levels and how application of music therapy can be used as an intervention in the surgical area. The study presents documentation on how music plays a significant role when used in surgical procedures emphasizing on both music as well as therapists in the therapeutic intervention.

Groene (1993) found that Alzheimer patients seated longer for music sessions than reading sessions under all conditions. Participants were randomly assigned to either music attention or reading attention group. Seating proximity sessions were recorded in a videotape. An MMSE was administered at both pre and post sessions and finally result analyses were made.

Palakanis, Jhon W, Brian and Charles L. (1994) found that patient's acceptability and compliance with the screening protocols of sigmoidoscopy gets affected due to their increased level of anxiety, this in turn results into prolonged or aborted procedures. They found music therapy to be a safe, economical and effective no pharmaceutical anxiolitic agent in reducing their anxiety levels.

Hanser and Thompson (1994) found that those older adults who were experiencing symptoms of depression, anxiety and distress, when assigned to musical conditions performed better than the controls on standardized tests of depression, mood, self-esteem and distress. The improvements were found by the clinicians to be significant and were found to follow-up a period of over nine months. 
Henry and Linda (1995) found that music therapy plays a significant role in decreasing the level of anxiety and pain among critical care patients. Even when they are suffering from some severe illness, use of music therapy helps in reducing their level of pain and anxiety. Thus more researches on ways of using music therapy as a complementary part of medicinal treatment was suggested.

Hammer (1996) found that Guided Imagery Music relaxation therapy and technique benefits patient with chronic stress and anxiety disorder, by reducing their level of perceived stress and anxiety. The study took place at a rehabilitation facility to know the effect of Guided Imagery Music therapy on the perceived levels of stress and anxiety among subjects.

Watkin and Gwendolyn (1997) found that music therapy can be of use in wide range of clinical settings comprising of patients suffering from migraine headaches, gastrointestinal ulcers and hypertension cardiovascular diseases. Their findings suggested that music therapy can be of great help in reduction of stress, anxiety levels, blood pressure and plasma stress hormone levels.

Chlan (2000) found that music therapy can be used as a nonpharmachologic intervention in the care of patients supported by mechanical ventilation. He gave a theoretical base of music therapy for anxiety reduction and relaxation, and highlighted the research testing intervention in such patients. Use of music therapy in critical care for nursing patients and promote a healing environment for them.

Nayak, Sangeetha, Barbara,Wheeler, Shiflett, Sameul and Sandra (2000) found that music therapy was a complementary therapy for social functioning and participation in rehabilitation, also tends to improve mood levels during the process of rehabilitation. Music therapy techniques acted as an aid for improving social interaction and mood after stroke or traumatic brain injury.

Prensner, Yowler, Charles, Smith, Steele, Louise and Richard (2001) found that music intervention in the management of pain among patients with primary issues of burn care has been helpful. It has acted as a means of self exploration and normalizing the environment. Music therapy for burned patients is adapted under several protocols, to meet the specific needs of the burned patients.

Hilliard (2001) found that music therapy helped in reducing grief symptoms among grieving children, and also improved their mood and behavior. All the subjects were made to participate in a battery of psychometrics which measures mood, behavior and grief symptoms for both pretests and posttests. 
Almerud and Petersson (2001) found that music therapy can be beneficially applied by the intensive care nursing staff as a non-pharmacological intervention. Music therapy was provided to patients who were temporarily on respirator in an intensive care unit, in order to measure the relaxation affect on patients.

Covington and Holly (2001) found that music therapy helps individuals suffering from psychiatric disorders to strengthen their coping skills of communication, self expression and socialization. Any struggle with poor communication skills, coping skills, self-expression and socialization leads to dysfunctional cognitive, emotional and social behavior. Therapeutic music sessions turned out to be a non-invasive to strengthen these skills and effect behavior change.

Browning (2001) found that women consider music therapy as a supportive and helpful coping strategy during labor. They used music therapy as a medium to distract themselves during labor from the pain that the experience and the results were satisfactory. Thus use of music therapy can be of help in prenatal preparations and pain and stress management during labor and child birth.

McCaffrey and Freeman (2003) found that music therapy among elder population lead to a reduction of chronic ostereoarthritis pain. The effect was more positive on those who listened to the music as compared to those who sat quietly without listening to music. Chronic pain among elder people acts as an obstacle for them to function properly and stay independent. Effect of music helps them feel motivated, elevates their mood and instills a sense of control among the elders.

Crowe and Rio (2004) found the use of technology in the music therapy practice in various settings, by providing the therapists, educators and clinicians an accurate and specific accounts of the types of technologies that can be brought in use. Technology application in music therapy was organized as follows: (a) electric/electronic musical instrument, (b) recording technology (c) adapted musical instruments, (d) computer applications (e) assistive technology for the disabled, (f) medical technology (g) technology based sound healing practices.

Sutoo and Akiyama (2004) found that music leads to an increase in the level of Calcium/CaMdependant DA synthesis in the brain. This in turn reduces blood pressure. Music affects the functioning of the brain through dopaminergic neurotransmission and thus it would be of great help in rectification of symptoms in various diseases which involves DA dysfunctions.

Urakawa and Yokoyama (2005) found that increased sympathetic nerve activity and promotion of such response can be achieved by synchronization of preferred music with the activated physical response, after an exercise in which sympathetic physical response is dominant. Hence 
Application of Music as an Element in Art Therapy, on Cancer Patients, Leads to Emotional Conflict Resolution and Body Healing. With an Insight into Sufi Music, Ancient Music Therapies, their Healing History as Well as Properties

combining music with exercise has been regarded as a good idea as it is enjoyable in terms of mood and often promotes physiological excitation thus enhancing physical activation.

Wong and Smith (2006) found that Complementary and Alternative Medicinal therapy (music therapy being one of the crucial therapies among them) used among children diagnosed with autism spectrum disorder was higher when compared to its use among the general population. Parents felt that use of such therapies along with other biologically based therapies were found more than seventy-five percent to be beneficial.

Hadley (2006) found that music therapy holds great potential as an agent for consciousness raiser, life changer and confidence builder among women who have been survivors of domestic violence. It can be used to empower them and help them find a voice for the process of internal emotional healing. It helps them work better on their self growth, enhancement and well-being.

Maratos, Wang and Crawford (2008) found that that those patients who were randomized to music therapy experienced greater reduction in symptoms of depression than those in standard care condition. Depression is considered to be a disorder which is highly prevalent and leads to low quality of life as well as reduced social functioning. Music therapy was found to play a significant role in reducing the symptoms of depression to an acceptable level.

Yeuh Chang, Hey Chen, Feng Huang (2008) found that listening to music by women during their pregnancy lead to reduced depression, stress and anxiety. It was encouraged because of being a cost-effective method and provides quantifiable psychological benefits. The t-tests showed that music brought a significant decrease in the perceived stress scale, state scale of the state anxiety inventory and Edinburgh Postnatal Depression scale.

\section{AN INSIGHT INTO INDIAN MUSIC THERAPIES, ITS HEALING HISTORY AS} WELL AS PROPERTIES

Indian musical ragas have a significant importance worldwide in connecting hearts of millions through communication of emotions and expression of self in the most artistic manner one can creatively think of. Raga is the sequence of selected notes in a selective combination that lends an appropriate mood or emotion. Raga depending on its nature can intensify or induce strong emotions like joy, sorrow, peace or violence, thus it forms the basis for all musical applications. It is believed that a raga holds the potential to capture and communicate a range of emotions within certain rhythms and melodies. Playing and listening to ragas works as medicines and thus have an impact on certain ailments.

The Raga system is believed to have been originated from the Rig Vedic period preceding the prehistoric Indus Valley Civilisation. The Vedic hymns of that period were meant for Yajnas 
called Yajur Vedas and the hymns used for singing were known as Samvedas. Music was formed out of rig Vedas, were set in to various tunes and tones known as Samaganas and then finally was cultured for some religious motive or spiritual purpose. People during those ages strongly believed into the science of music and that music has the capacity of healing and enhancement of overall environment. The Vedas and Upanishads had most of the sounds and rhythms as they were found to be a source of healing as well as upliftment. The tunes used in samaganas were five, six and seven and they were harmonized into tunes,speech and rhythm. From samagana the seven notes of music has been evolved, which formed the raga system in India and is now known as worldwide music. After Vedic Music, evolution of Gandharva and Marga music took place and finally "desi” and regional music were formulized. The raga system followed today in India is however much more systematic and scientific having seventy-two mela karta (parent raga) scheme, which was devised by Venkatamakhi with various combinations and permutations of swaras under janya and mela karta classification in south Indian music and ten basic that as or melas in North Indian Music which has been found to contribute to the development and enrichment of raga system. Since time and ages it has been considered to be a fact and soon with developing scenario neural researches proved that the seventy-two ragas control seventy-two nerves in a human body.

Nada yoga is an ancient healing practice and the word "Nada" in Indian philosophy means sound vibration. It is considered to be the universal force and not just a resonance. The entire universe is made up of sound vibrations which has been classified into two types, Ahata (struck sound) and Anahata (unstruck sound). The Nada is composed of divine universal energies and the source for this divine energy is "Anahata Chakra" situated in the central region of the heart. The Primeval sound "Aum" is known to be the creator of the universe and the chakra or the spiritual center is known to be it's seat in the heart region.

The ancient Hindus have relied on music for its curative properties. Vedic chants in praise of Gods were believed to have immemorial cure for various disharmonies of an individual and his or her environment. Through historical records we get to know that Haridas Swami who was guru of the famous musician Tan Sen in Akbar's time, had recovered a queen of the emperor with one of his raga. The greatest composers of classical music in India, Bach, Mozart and Beethoven known as "Musical Trinity" who were curiously contemporaries of the "Trinity of Western Classical Music, were known to be sensitive towards acoustical energies. It is believed that Saint Thyagaraja through his Bilahri composition “Naa Jiva Dhaara”, brought

a dead person back to life. Navagriha Kriti by Muthuswamy Dikshitar, was believed to cure stomach ache and Duru Sugu, a composition by Shyama Sastry was a music used to pray for good health. 


\section{INDIAN RAGAS AND THEIR EFFECT ON BODY CHAKRAS}

When the swaras are sung in an ascending order it is known as "Arohanam" and when they are sung in descending order it is known as "Avarohanam". It is interesting to know how the seven swaras comprising a raga can have deep rooted effects in our body through their influence on our seven spiritual body chakras. These seven chakras are considered to be vital to our health. When an individual feels sick or is diseased then it's often because of the hampering of the chakras by some negative feelings. Though today's modern medicinal means cannot detect their existence within our body, but they are believed to be the vital energy center in our body. Here are the ways how the seven ragas influence the seven spiritual chakras in a human body:

SA: Sa shajdam is the basic of all musical notes, responsible for the interpersonal relationship of the notes that define a raga, as well as the relationship of these notes to the basic note (adhaar shajdam). It increases the vibration of Mooladhar chakra important for survival. Kundalini shakti is known to exist at the tip of the backbone in a coiled form, increased vibration of mooladhaar chakra raises kundalini shakti. This when awakened moves through the shushumna nadi (spinal cord) upwards towards the Sahasrara chakra where lord Shiva resides. For providing a smooth passage for the shakti to travel, each note is sung in an ascending order.

RI: Back, Swadhishtina Chakra

GA: Back Manipura Chakra

MA: Back Anahatha Chakra

PA: Back Vishudhi Chakra

DHA: Back Ajna Chakra

NI: Sahasrara Chakra

SA: Cosmos

After Arohanam, Avarohanam is sung in descending order to connect and reinforce the Shiv shakti and Cosmos at the sahasrara chakra. The combined shiv shakti then flows through the front chakras and increases the divine energy of the corresponding chakras. Singing of the swara garland makes individual gain energy from the cosmos as well as mother earth.

Ragas and their Effects in Healing

Kafi Raga: Evokes a deep, soothing and humid mood.

Raga Pooriya Dhansari (Hamsanandi-Kamvardini) : Known to prevent acidity and evokes a sweet, heavy, deep, cloudy and stable state of mind.

Raga Bageshri: evokes a feeling of calmness, depth, stability and darkness, also is used for curing Diabetes and Hypertension.

Raga Mishra Mand: Evokes a sweet and pleasant feeling. Makes one feel calm and composed. Raga Darbari (Darbari Kanada) : Used for recovering from tension. 
Application of Music as an Element in Art Therapy, on Cancer Patients, Leads to Emotional Conflict Resolution and Body Healing. With an Insight into Sufi Music, Ancient Music Therapies, their Healing History as Well as Properties

Raga Abhir-Bhairav (Chakravakam): Used to sustain chords and bring down blood plessure. Raga Bhupali and Todi: Cures high blood pressure. Raga Malkauns and Raga Asawari (natabhairavi) : Cures low blood pressure.

Raga Bihag, Bahar (Kanada) and Kafi and Khamaj: Helps recover from insomnia and other sleep disorders.

Raga Tilak-Kamod (Nalinakanti), Hansdhwani,Kalavati,Durga (Suddha Saveri) : Causes a very pleasant feeling on the nerves.

Raga Malhar: Asthma and Sunstroke.

Raga Bhairavi: Relief from severe cold phlegm, Sinus, Toothache, T.B and Cancer.

Raga Todi, Jayawanti and Poorvi : Relief from Cold and Headache.

Raga Hindol and Marwa: Blood Purification.

Raga Kharahara Priya: Curative for distress, worries, heart diseases, tension, neurosis, nervous irritability and strengthens mind.

Raga Shivaranjani: For memory problems.

Raga Hindolam and Vasantha: relief from gastritis, blood pressure, vatha roga and purifies blood.

Raga Saranga: Cures Pitha Roga

Raga Punnagavarali, Sahana: controls anger as well as violence.

Raga Natabhairavi: cures headache and psychological disorder.

Raga Dhwijavanthi: quells sicorders of the mind and paralysis.

Raga Kapi: To overcome depression,anxiety and absent mindedness.

Raga Ganamurte: Helpful in diabetes.

Raga Ranjani: Cures Kidney disorders.

Raga Shanmukhpriya: Instills courage in one's mind.

Raga Rathipathipriya: Adds vigor and strength to a happy wedded life.

Raga Hameerkalyani: Relaxes tension and brings down blood pressure level.

Raga Sindhubhairvi: For happy and healthy mind and body.

Raga Brindavana Saranga: Knowledge, success, wisdom, joyfulness and greater energy.

Raga Mohana: Cures migraine headache.

Raga Ananda Bhairvi: Cures stomach pain, Kidney problem and controls blood pressure.

Raga Charukesi Kalyani, Sankarabharanam and Chandrakauns: Used for heart Ailments.

Raga Amrutavarshini: Alleviates heart related diseases.

Raga Madhyamavati: Cures pain in legs and hands, paralysis and giddiness.

Raga Reethigowla: Bestows direction to one who seeks it.

Music Therapies Practiced In India

With development in human kind, there has been more variety of music that has been introduced on the ground of emotional healing and mental positivity enhancement. Some of them are Sufi songs (songs in which an individual refers to the almighty as his/her beloved), Qawalis ( songs 
sung in praise, devotion and love for almighty in dargas), Bhajans (songs sung in appreciation, gratitude, love and praise of God in temples and rituals or during worships), instrumentals(both classical and western) etc. But even then the classical Indian Ragas have been able to occupy a significant place for itself in the field of music therapy. Even today large number of therapist recommend listening to these ragas for over a good period of time and also makes their practical application for achieving most desired results.

These music therapies have turned out to be a blessing for their multiple advantages that they hold in relation tot heir usage. These therapies can be put to use in any setting much conveniently and promises no side-effects resulting from their application. Therefore more and more people are giving it much preference in the field of complementary and alternative therapies for body and emotional healing. Music has been considered as a tool for catharsis as well. It has been observed to hold immense potential to heal emotions in the process of wenting out, but with the discovery of its properties to heal specific body and emotion related issues makes it all the more most widely used therapy among therapists and care givers.

Music therapy practices in India have been growing since it's evolution and till date has been performed successfully with immense satisfactory and desired results. More development in this field in anticipated with more discoveries taking place in these genres of music. Therapists and researchers are working to find several other new interventions which can be brought in for facilitating a sensible growth in the usage of music therapies among more and more population. India is making an effort to join in with many other countries and explore the magnificent art of healing that music practically holds and can be very well brought into use by both therapist as well as caregivers on their respective clients and patients.

Especially in a country like India, where people seek for such therapies which are economical as well as effective, music therapy is predicted to work wonders for such hopeful population through fulfillment of their maximum desired expectations. Hence such complementary therapy is expected to be one of the conventional therapies used in India in time to come, for the purpose obtaining optimum and desired healing results.

\section{DISCUSSION AND CONCLUSION}

The current research analysis has been done with an aim to study the role of Music Therapy in Cancer and Body Healing, with an insight into the Ancient Indian Music Therapies, their healing history and properties. The research analyses are based on secondary data and historical information. Studies show that music therapy plays a significant role in the process of healing and health enhancement among cancer patients as well as those who suffer from some other health related issues. Music therapy has been in use since Vedic period and till date has been performing well in the process of healing patients. The origination of music during early ages has 
been based on various spiritual concepts which are followed and well accepted by today's researchers and therapists. Music therapy was provided in historical times through various Ragas that were a combination of different notes formed by seven swaras. It is believed that the entire universe has been created out of sound vibrations which are of two types, Ahata (struck sound) and Anahata (unstruck sound). It is believed and researchers have studied that the seven swaras affect the seven spiritual chakras existing at the back on the spinal cord. The primeval sound of "Aum" is said to be the creator of the universe. There are multiple ragas which causes healing effect on multiple body related issues.

Among cancer patients, music therapy has been found to have helped them enhance their ways of living, personality and wellbeing. Their level of depression, anxiety, stress and pain is found to get reduced and more vigor and positive attitude is developed. Helps them in eliciting their emotions and feelings in a better manner also supports them in their process of coping with the situation and its demands. Their level of immunoglobulin is increased and their level of cortisol is decreased which is considered to be a positive sign. Those who listened to live music were found to respond better than those who listened to tape recorded music. Reduces pain among women who undergo radical mastectomy and reduces distress among those who are to undergo curative radio therapy. Earlier their has been stereotype thinking over the introduction and application of music therapy in the medical process of healing among patients, but with time, studies and researches it gained grounds in the clinical setups and was actually found to work wonders in case of those suffering from cancer.

Music therapy played a significant role in the process of body healing among patients apart from those who suffered from cancer. It was found to reduce blood cholesterol level, high blood pressure, cure low blood pressure, cure aches, enhance neurological abilities, pregnancy, rehabilitation, emotional healing etc. It plays a vital role in the enhancement of overall wellbeing of individuals. It is the most economical and a side effect free therapy and thus is most preferred one among the patients, for their any body related issues. It increases sympathetic nerve activity and the level of calcium CAM-dependant DA synthesis in the brain. It is the most widely used complementary and alternative medicine for the process of healing, holding an assurance to provide desired results the most cost-effective and anxiolitic manner, with absolutely no sideeffects.

Music therapy will play a significant role in the field of spiritual healing as well. It holds it's derivation from various spiritual concepts which are well accepted not only in India but also various other nations. And since the usage of therapies to a good extent depends on the susceptibility of the patients, a large number of population in India is expected to get influence by it's properties of spiritual healing. Thus we'll get to see he usage of music therapy being expanded to greater horizons than just clinical and counseling setups. With more and more 
Application of Music as an Element in Art Therapy, on Cancer Patients, Leads to Emotional Conflict Resolution and Body Healing. With an Insight into Sufi Music, Ancient Music Therapies, their Healing History as Well as Properties

people developing faith in the healing art of music, more possibilities of music becoming a conventional therapy in certain healing processes is likely to take place. Music therapy for its spiritual healing properties has been considered as a dependable source of meditation as well.

Now that we get to see and observe in the present scenario, that music has gained grounds on various scientific reasoning as well, one can always predict its development as a complementary medicine in the process of treatment of various patients. But with such innovations would require mature brains to coordinate, cooperate and work efficiently in managing its apt usage in any particular set-up. The efficiency of any therapy depends on the efficiency of the process in which its application has been made. Thus the results of the music therapy and the efficiency of its process of application are in direct or rather in positive relation to one another. For better results, results the process of therapy should be equally good.

Music therapy acts as a catalyst or stimulus for an individual's emotional experiences. It helps in the intensification of the sense of emotions and thus results in better analysis of emotional distress or any other related issues. Music therapy has been thus given preference in the process of behavioral analysis or rather technical development of behavior of an individual.

\section{KEY LEARNING}

(Indian Music therapy history, healing properties and role in body healing)

- Music therapy has been in use in India since the Vedic period and Indus Valley Civilization.

- $\quad$ Since then great musicians have made significant contributions in the field of music as a healing art.

- $\quad$ Music therapies affect the seven spiritual chakras of the human body.

- $\quad$ The seven respective Swaras (Sa,Ri, Ga, Ma, Pa, Dha and Ni), causes an individual effect on the seven respective chakras, starting from the Mooladhar chakra and ending at Sahasrara chakra.

- Shakti is said to be awakened through Arohaman (ascending order of swaras) and the shiv shakti and cosmos engery reinforces at the sahasrara chakra through Avrohaman (descending order of swaras).

- $\quad$ The energy from mother earth and cosmos energy travels through the spinal cord,entering from the mooladhar chakra, when musical notes or swaras are sung.

- $\quad$ Primeval sound "Aum" is said to be the creator of the universe.

- $\quad$ It seats in the heart region.

- "Nada" means sound vibrations.

- $\quad$ The entire universe is made up of sound vibrations divided into Ahata(struck sound) and Anahata (unstruck sound).

- $\quad$ There are seventy-two parent ragas known as mela-kartas in south-Indian music. 
- $\quad$ There are ten basic thatas in north-Indian music

- $\quad 72$ notes of music is said to control 72 nerves in the human body.

- $\quad$ Music therapy helps in healing various psychological, emotional as well as body related problems.

- $\quad$ Music helps in reducing anxiety, depression and stress among patients.

- It helps patients of Alzheimer's to be seated in a particular place for a longer period of time, hence evoking positive responses among them.

- Helps in bringing down level of anxiety among patients who are to undergo Sigmoidoscopy.

- Is a nonpharmaceutical anxiolitic agent.

- $\quad$ Cures pain, aches, enhances wellbeing, self esteem and controls mood fluctuations.

- Holds no side effects and increases the level of calcium CAM-dependant DA synthesis in the brain.

- $\quad$ Beneficial in pregnancy

- $\quad$ Beneficial for those who suffer domestic violence.

- $\quad$ Increases sympathetic nerve activity.

- $\quad$ Helps in the process of rehabilitation.

\section{KEY LEARNING}

(Role of Music therapy in Cancer Healing)

1. Less tension, anxiety, depression and more vigor.

2. Serves as a mode of eliciting feelings and in the process of coping.

3. Non threatening form of communication and an outlet for emotional healing.

4. Increases their level of immunoglobulin and decreases cortisol level.

5. Reduces emotional distress of those who undergo curative radiotherapy.

6. Turned out to be a creative, complementary and alternative therapy.

7. Cures pain of women who undergo radical mastectomy.

8. Those who listened to live music showed better level of improvements than those who listened to tape-recorded music.

9. Guided imagery and music have been found to make significant contributions in the life of those who suffer from cancer.

10. Promotes healthy living and enhances their wellbeing.

11. Conscious expansion.

12. Analyses the importance of creativity in the process of individuation.

13. Effective results can be obtained through effective cooperation between the patient and the caregiver.

14. Strengthens the right hemisphere of the brain.

15. Influences the immune system of the body. 


\section{REFERENCES}

Aditya Bardia, Debra L. Barton, Larry J. Prokop, Brent A. Bauer and Timothy J. Moynihan; Efficacy of Complementary and Alternative Medicine Therapies in Relieving Cancer Pain: A Systematic Review; 2006.

Barbara J. Crowe, Robin Rio ; Implications of Technology in Music Therapy Practice and Research for Music Therapy Education: A Review of Literature; 2004.

Burns SJ, Harbuz MS, Hucklebridge F, Bunt L ; Alternative Therapies in Health and Medicine;2001.

Caryl Ann Browning; Using Music During Childbirth; 2001.

Chlan, Linda; Music Therapy as a Nursing Intervention for Patients Supported by Mechanical Ventilation; 2000.

Clare O'Callaghan, Fiona McDermott; Music Therapy's Relevance in a Cancer Hospital Researched Through a Constructivist Lens; 2004.

Cook, Janet; Music as an intervention in the oncology setting; 1986.

Covington, Holly; Therapeutic Music for Patients with Psychiatric Disorders; 2001.

Debra S. Burns; The Effect of the Bonny Method of Guided Imagery and Music on the Mood and Life Quality of Cancer Patients;2001.

Den'etsu Sutoo, Kayo Akiyama; Music improves dopaminergic neurotransmission: demonstration based on the effect of music on blood pressure regulation; 2004.

Edzard Ernst; Complementary therapies in palliative cancer care; 2001.

Effect of music therapy on mood and social interaction among individuals with acute traumatic brain injury and stroke; 2001.

Eric G. Waldon; The Effects of Group Music Therapy on Mood States and Cohesiveness in Adult Oncology Patients; 2001.

Helen H. L. Wong, Ronald G. Smith; Patterns of Complementary and Alternative Medical Therapy Use in Children Diagnosed with Autism Spectrum Disorders; 2006.

Henry, Linda L. ; Music Therapy: A Nursing Intervention for the Control of Pain and Anxiety in the ICU: A Review of the Research Literature; 1995.

Kayoko Urakawa, Kazuhito Yokoyama; Music Can Enhance Exercise-Induced Sympathetic Dominancy Assessed by Heart Rate Variability; 2005.

Kerry C. Palakanis., Dr. John W. DeNobile, Charles L. Blankenship ;Effect of music therapy on state anxiety in patients undergoing flexible sigmoidoscopy; 1994.

Lucanne Magill Bailey ; The Effects of Live Music versus Tape-Recorded Music on Hospitalized Cancer Patients; 1983.

Malgorzata Monika Stanczyk; Music therapy in supportive cancer care;2011.

Mei Li, Yan, Zhou, Dang, Whang and Zhang; effects of music therapy on pain among female breast cancer patients after radio mastectomy; 2010.

Mei-Yueh Chang, Chung-Hey Chen and Kuo-Feng Huang; Effects of music therapy on psychological health of women during pregnancy; 2008. 
Application of Music as an Element in Art Therapy, on Cancer Patients, Leads to Emotional Conflict Resolution and Body Healing. With an Insight into Sufi Music, Ancient Music Therapies, their Healing History as Well as Properties

Nayak, Sangeetha; Wheeler, Barbara L.; Shiflett, Samuel C.; Agostinelli, Sandra;

Prensner, Jennifer ,Yowler, Charles J. ,Smith, Lori F., Steele, A. Louise, Fratianne, Richard; Music Therapy for Assistance with Pain and Anxiety Management in Burn Treatment; 2001.

Robert William Groene; Effectiveness of Music Therapy, 1:1 Intervention with Individuals Having Senile Dementia of the Alzheimer's Type; 1993.

Russell E. Hilliard; The Effects of Music Therapy-Based Bereavement Groups on Mood and Behavior of Grieving Children: A Pilot Study; 2001.

Ruth McCaffrey ,Edward Freeman; Effect of music on chronic osteoarthritis pain in older people; Article first published online: 20 NOV 2003

Sofia Almerud, Kerstin Petersson; Music therapy—a complementary treatment for mechanically ventilated intensive care patients; 2001.

Susan E. Hammer; The Effects of Guided Imagery Through Music on State and Trait Anxiety; 1996.

Susan Hadley; Feminist perspective of music therapy; 2006.

Suzanne B. Hanser ,Larry W. Thompson; Effects of a Music Therapy Strategy on Depressed Older Adults; 1994.

Updike, Phyllis; Charles, David; Music Rx: Physiological and Emotional Responses to Taped Music Programs of Preoperative Patients Awaiting Plastic Surgery; 1987.

Vicki A. Moss; Music and the Surgical patient, the Effect of Music on Anxiety ; 1998.

How to cite this article: S Mukherjee (2016), Application of Music as an Element in Art Therapy, on Cancer Patients, Leads to Emotional Conflict Resolution and Body Healing. With an Insight into Sufi Music, Ancient Music Therapies, their Healing History as Well as Properties, International Journal of Indian Psychology, Volume 3, Issue 3, No. 9, DIP: 18.01.159/20160303, ISBN: 978-1-365-13820-1 